\title{
Application of intramolecular carbonyl-ene reaction towards the synthesis of idarubicinone scaffold
}

\author{
Shyam Basak, Sutapa Ray, and Dipakranjan Mal* \\ Department of Chemistry, Indian Institute of Technology Kharagpur, Kharagpur 721302, India \\ E-mail: dmal@chem.iitkgp.ernet.in
}

Received 11-02-2017

Accepted 03-20-2018

Published on line 04-16-2018

\begin{abstract}
Intramolecular aromatic carbonyl-ene reaction (ICE) ${ }^{\dagger}$ has been combined with a modified Hauser annulation to offer a facile access to the tetracyclic idarubicinone core. The required key dihydroxyanthraquinone aldehyde precursor was assembled in one step by modified Hauser annulation of a functionalized benzoquinone. Its ene reaction in the presence of $\mathrm{SnCl}_{4} \cdot 5 \mathrm{H}_{2} \mathrm{O}$ directly led to the formation of idarubicinone core. Also described are an unprecedented thermal cascade involving a thermal ICE en route to $\alpha$-naphthols, and exploratory studies on model elaboration of anthracycline A rings.
\end{abstract}

'By "aromatic carbonyl-ene reaction" we mean those reactions in which an aromatic ring is embedded in the transition state of a carbonyl-ene reaction.

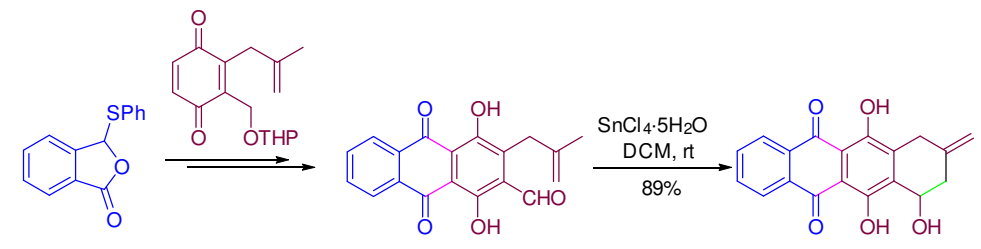

Keywords: Intramolecular, carbonyl-ene, tetralin, anthracyclines 


\section{Introduction}

The type-II intramolecular carbonyl-ene reaction (ICE) shown as eqn. 1 in Scheme 1 is an established process in the organic synthesis. ${ }^{1-2}$ It has allowed total synthesis of many bioactive natural products. ${ }^{3-8}$ However, its aromatic version (eqn. 2, Scheme 1) discovered in 1983 by Hauser and Mal in conjunction with their work on the total synthesis of $( \pm)-\gamma$-citromycinone, has been limited to anthracyclines. ${ }^{9-15}$ It is a significant advancement in the area of ICE, in view of its success despite the conformational constraint imposed by a benzene ring. Furthermore, the placement of the benzylic hydroxyl group in the product obviates the problems of benzylic bromination under radical conditions on a large scale. ${ }^{16,17}$ However, its application to simple naphthalenes and anthracenes are problematic due to the susceptibility of the incipient ene products to aromatization. In a recent report ${ }^{18}$ from our laboratory, we have demonstrated that the ICE can be manoeuvred to prevent aromatization of the ene products, thus permitting synthesis of non-aromatized ene products i.e. hydroaromatic products. We also included development of a synthesis of ortho methallylbenzaldehydes based upon Suzuki coupling reactions.

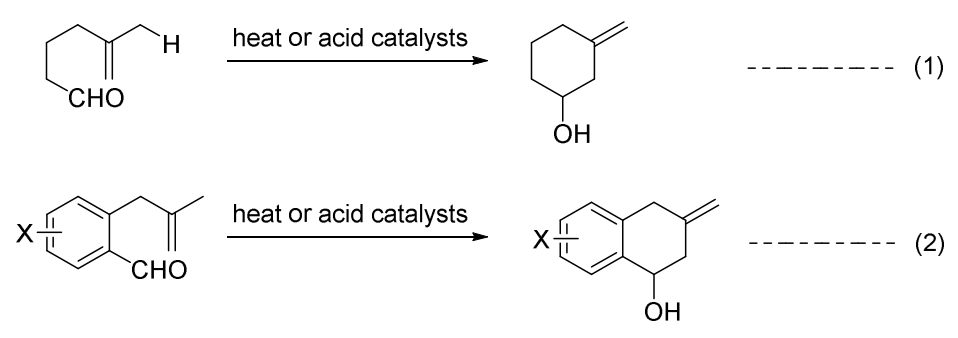

Scheme 1. Type II carbonyl-ene reaction.

In seeking an application of the aromatic ICE in developing a simpler route to anthracyclines 1-5 (Figure 1), the most widely used anticancer drugs ${ }^{19-22}$, we chose to assemble the tetracycline framework of idarubicinone (3) as shown in Scheme 2. The salient features of the retrosynthesis are i) modified Hauser annulation of 6 and 7 to assemble dihydroxy intermediate $\mathbf{8}$ and ii) its direct use as the ene substrate to produce tetracyclic intermediate 9. It may be mentioned that the reactivity of the 1,4-dimethoxy analog of quinone 8, prepared by Claisen rearrangement, was examined towards the aromatic ICE. ${ }^{23}$ No further elaboration was attempted probably due to the problem with deprotection of the aromatic methoxy groups without affecting the A-ring. In devising the synthesis of $\mathbf{3}$, we, therefore, envisioned investigations with dihydroxy intermediate $\mathbf{8}$. It was anticipated that the corresponding ene product i.e. $\mathbf{9}$ would be sufficiently stable due to intramolecular hydrogen bonding for further elaborations without resorting to a deprotection.

In this study, the potential of the aromatic ICE approach in creating an asymmetric centre at C7 position of anthracyclines is proposed. Asymmetric epoxidations or hydroxylations or nucleophilic additions can be invoked for further elaborations, as indicated in Scheme 2. Asymmetric dihydroxylation of the ene product 9 is proposed to give $\mathbf{1 0}$ and then $\mathbf{3}$. In the second approach, tetracycle $\mathbf{1 1}$ is expected to produce compounds $\mathbf{1 2}$ and 13 and $\mathbf{3}$ (Scheme 2). 


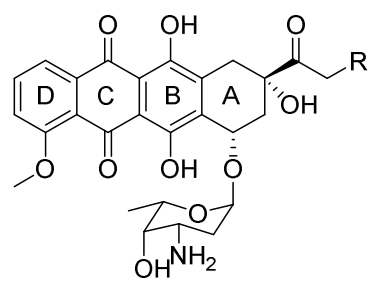

$\mathrm{R}=\mathrm{H} \quad$ Daunorubicin (1)<smiles>COc1cccc2c1C(=O)c1c(O)c3c(c(O)c1C2=O)C[C@@](O)(C(=O)CO)C[C@H]3OC1CC(N)C(O)C1(N)CO</smiles>

Epirubicin (EPI) (4)

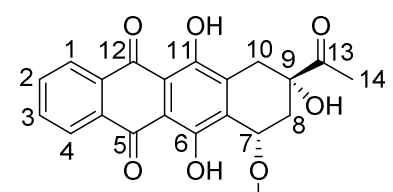

$$
{ }_{\mathrm{OH}^{\mathrm{NH}}}
$$

Idarubicin (IDA) (3)
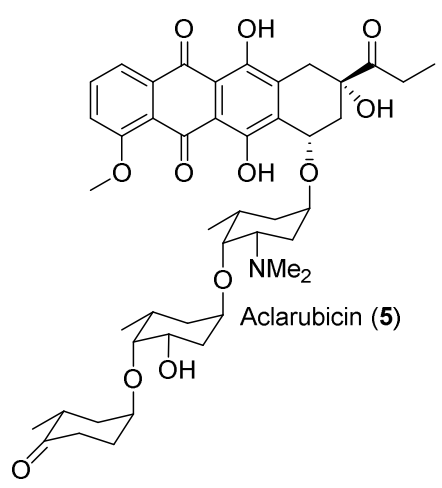

Figure 1. Selected clinically used anthracyclines.

Retrosynthetic approach 1<smiles>C=CC(=O)[C@]1(O)Cc2c(O)c3c(c(O)c2C[C@H]1O)C(=O)c1ccccc1C3=O</smiles>

idarubicinone (3)<smiles>C=CC(C)[C@]1(O)Cc2c(O)c3c(c(O)c2C(=O)c2ccccc2C1=O)C(=O)c1ccccc1C3=O</smiles>

10<smiles>C/C=C1/Cc2c(O)c3c(c(O)c2[C@@H](O)C1)C(=O)c1ccccc1C3=O</smiles>

9

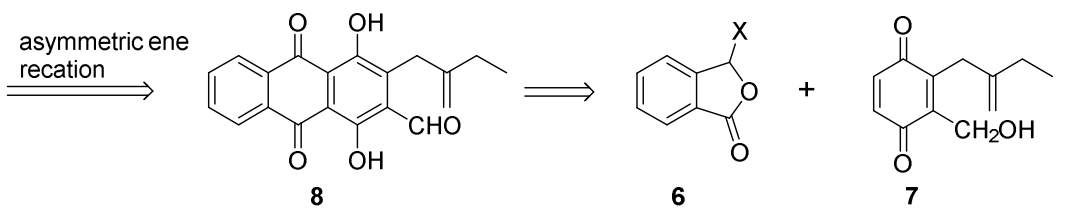

Retrosynthetic approach 2<smiles>C#CC1(O)Cc2c(O)c3c(c(O)c2C([O-])[C@@H](O)C1)C(=O)c1ccccc1C3=O</smiles>

Scheme 2. Retrosynthetic analysis of idarubicinone (3).

\section{Results and Discussion}

To perform a model study of idarubicinone (3) utilizing Hauser annulation, we first decided to synthesize the Michael acceptor 14 ( $c f$ 7, Scheme 2) (Scheme 3). This proposal stemmed from our previous study on the 
Hauser annulation of $p$-quinones for straightforward generation of 1,4-dihydroxyanthraquinone moieties. ${ }^{24}$ Accordingly, $O$-methallyl substrate 15 was prepared by selective $O$-methallylation of methyl 2,5dihydroxybenzoate with methallyl bromide in the presence of $\mathrm{K}_{2} \mathrm{CO}_{3}$ in acetone. Claisen rearrangement of 15 in boiling DMF gave two products 16 and 17 in 1:1 ratio. Appearance of a $2 \mathrm{H}$ singlet at $\delta 2.98$ and a $6 \mathrm{H}$ singlet at $\delta 1.50$ indicated the formation of $\mathbf{1 7}$, which was confirmed by its acetylation to compound 18 . Quinol 16 was methylated to 19 by reaction with $\mathrm{Me}_{2} \mathrm{SO}_{4}-\mathrm{K}_{2} \mathrm{CO}_{3}$ in acetone. Compound 19 was then reduced to alcohol $20^{25}$ by lithium aluminium hydride.

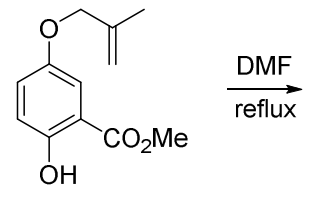

15

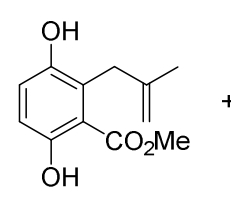

16<smiles>[R]Oc1ccc(O)c2c1CC(C)(C)OC2=O</smiles>

$17 \mathrm{R}=\mathrm{H}, 40 \%) \mathrm{Et}_{3} \mathrm{~N}$, acetyl chloride $18 \mathrm{R}=\mathrm{AC}$ DCM, $83 \%$

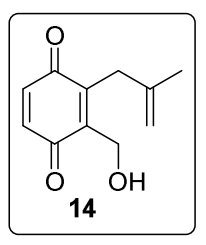

20

Scheme 3. Preparation of alcohol 20.

For an alternative route to $\mathbf{2 0}$, we examined the possibility of Claisen rearrangement of a related aldehyde precursor, and for the model study we chose aldehyde $21^{26}$ (Scheme 4).

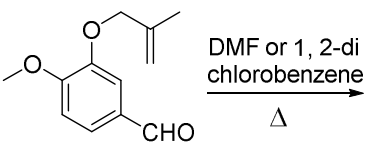

21

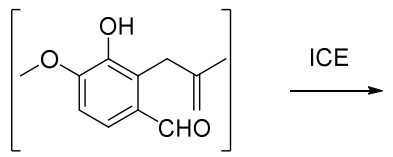

25

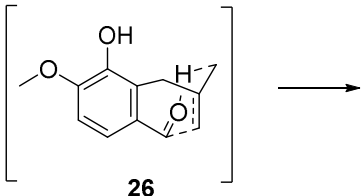

26
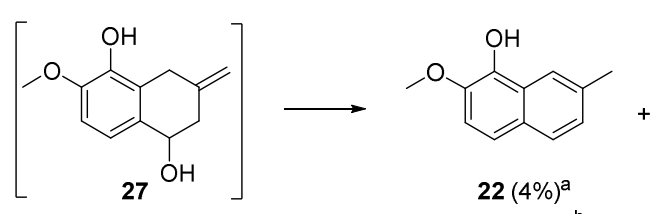

$$
(25 \%)^{b}
$$

a: DMF; b: 1,2-dichlorobenzene

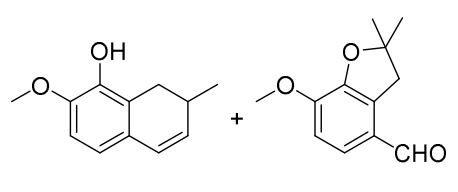

$23(7 \%)^{\mathrm{a}}$

$24(23 \%)^{\mathrm{a}}$

$(19 \%)^{b}$

Scheme 4. Thermal rearrangement of aldehyde 21.

When it was heated in refluxing DMF, the desired Claisen product was not obtained. Instead, the reaction resulted in formation of three products 22 (4\%), 23 (7\%) and $24^{27}$ (23\%) (Scheme 4). Formation of the naphthol $\mathbf{2 2}$ is accounted for by Claisen rearrangement of $\mathbf{2 1}$ to intermediate $\mathbf{2 5}$ followed by thermal ICE of $\mathbf{2 5}$ through $\mathbf{2 6}$ to $\mathbf{2 7}$, its dehydration and isomerization. The formation of $\mathbf{2 3}$ is very striking in that its formation must involve a reduction, since there is a decrease in the oxidation level. ${ }^{28}$ The probable mechanism is shown in Scheme 5, where DMF acts as the hydride donor. The structure of $\mathbf{2 3}$ is further confirmed by transforming it 
into its acetate. It may be noted that the product $\mathbf{2 3}$ was not obtained when the reaction was carried out in 1,2-dichlorobenzene. Formation of $\mathbf{2 4}$ is explicable in terms of its Claisen rearrangement of $\mathbf{2 1}$ followed a 5exo-trig cyclization of 25.

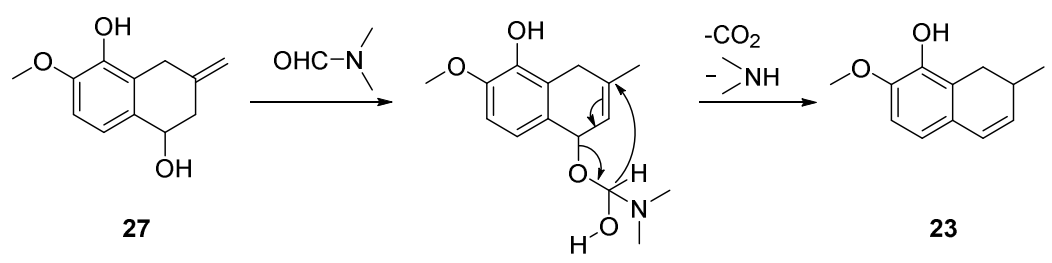

Scheme 5. Proposed mechanism for the transformation of $\mathbf{2 1}$ to 23.

In view of the problems with above Claisen rearrangement methodology, the synthesis of quinone 14 was started from the known aldehyde $28 .^{18}$ It was converted into quinone 14 in two steps. $\mathrm{NaBH}_{4}$ reduction of 28 gave alcohol 20 in $86 \%$ yield, which was oxidized with CAN to give 14 in $66 \%$ yield. It was then converted into THP ether 29 by treatment with DHP in dry DCM in the presence of a catalytic amount of PPTS (Scheme 6).

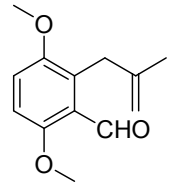

28

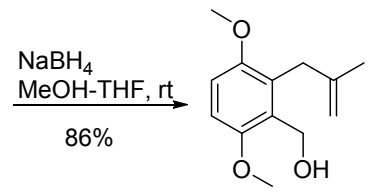

20

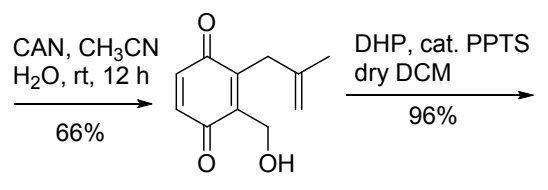

14

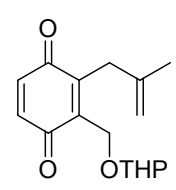

29

Scheme 6. Alternative synthesis of alcohol 20 and acceptor 29.

Both the quinones 14 and $\mathbf{2 9}$ were subjected to Hauser annulations with phthalide $30^{29}$. In the presence of LiOBu-t, the annulation ${ }^{24}$ of $\mathbf{3 0}$ with 14 gave annulation product $\mathbf{3 1}$, but in only $10 \%$ yield. Assuming that the free $-\mathrm{OH}$ in 14 interfered the annulation, we examined its protected form i.e. 29. Annulation of phthalide sulfide $\mathbf{3 0}$ with $\mathbf{2 9}$ under similar conditions gave dihydroxyanthraquinone $\mathbf{3 2}$ in $68 \%$ yield. Deprotection of $\mathbf{3 2}$ with PPTS-MeOH provided alcohol 31 in 76\% yield. PCC oxidation of $\mathbf{3 1}$ furnished aldehyde $\mathbf{3 3}$ in $69 \%$ yield. This was then subjected to ICE under a variety of conditions. Gratifyingly, we obtained tetracycle $\mathbf{1 1}$ as the sole product representing the core structure of idarubicinone (3) on treatment with $\mathrm{SnCl}_{4} \cdot 5 \mathrm{H}_{2} \mathrm{O}$. It was sufficiently stable to be purified by chromatography on silica gel (Scheme 7).

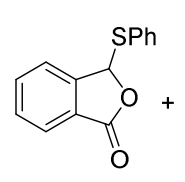

30

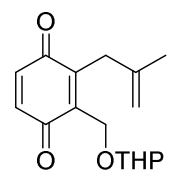

29

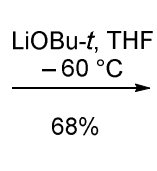

$8 \%$

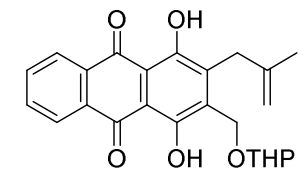

32

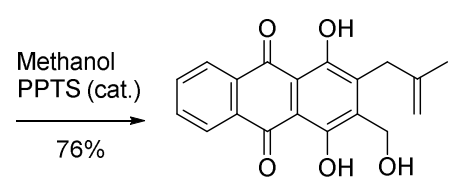

31

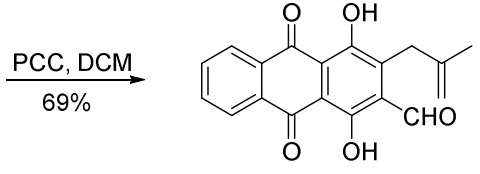

33
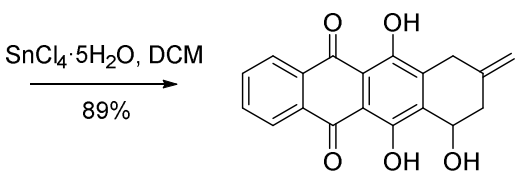

11 
Scheme 7. Synthesis of the tetracycle core of idarubicinone (3).

In parallel with the synthesis of the tetracycle 11, we performed few exploratory studies designed for functionalization of the $A$ ring with model ene derivative $34^{18}$ obtained from our previous work. Its NMOmediated dihydroxylation afforded triol 35 in 64\% yield along with diols 36 (15\%). Trans stereochemistry of 35 was confirmed by analysis of coupling constants of $\mathrm{C} 1$ hydrogen and comparing them with those for similar compounds. ${ }^{23}$ For 1,3-cis dihydroxy analogs, the corresponding coupling constants are less than $5 \mathrm{~Hz}$. It is noteworthy that the dihydroxylation produced only one diastereomer. Ozonolysis of the ene product $\mathbf{3 4}$ followed by treatment with triphenylphosphine did not furnish product 37. Instead, it produced aromatized product $\beta$-naphthol (38). But, its silyl derivative $\mathbf{3 9}$, prepared by silylation with TBSCl/imidazole gave $\mathbf{4 0}$ in $65 \%$ yield on ozonolysis. It is sufficiently stable for a chromatographic purification (Scheme 8).

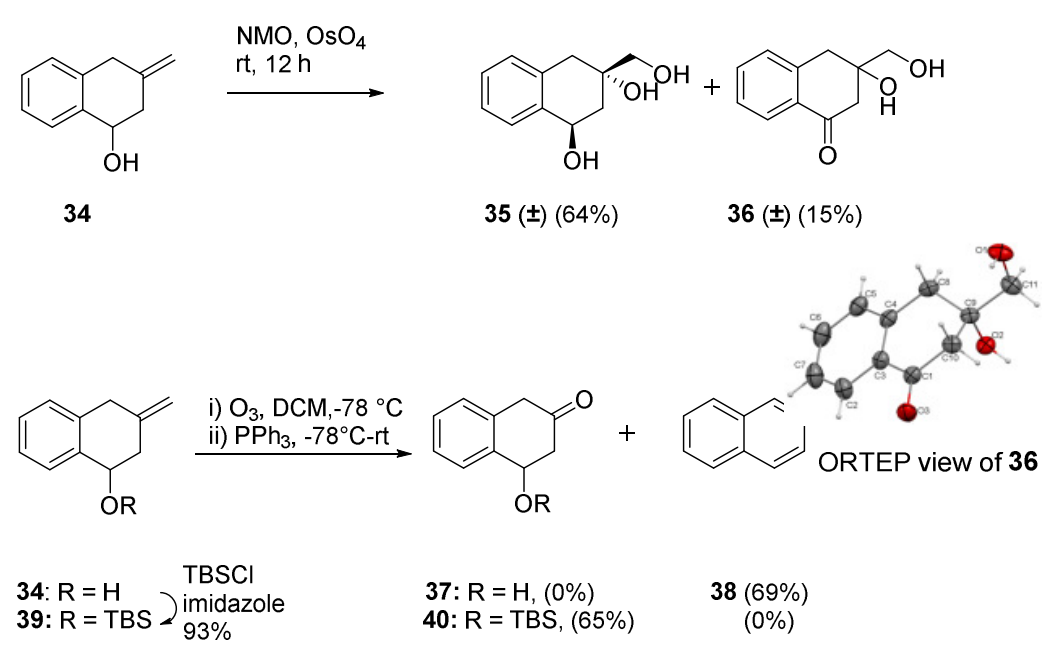

Scheme 8. Model study for functionalization of tetralin skeleton of anthracyclines.

\section{Conclusions}

The modified Hauser annulation i.e. annulation of phenylthiophthalides with 1,4-quinones was combined with aromatic carbonyl-ene reaction to pave a simple entry to tetracyclic core of anthracyclines. Noteworthy is the fact that tetracycle $\mathbf{1 1}$ with free phenolic - $\mathrm{OH}$ group is not very sensitive to aromatization, and it should be amenable to selective dihydroxylation.

\section{Experimental Section}

General. All reactions utilizing moisture-sensitive reagents were performed under an inert atmosphere. All solvents namely DMF, DCM, THF, MeOH etc. were dried prior to use, according to the standard protocols. Melting points were determined in open capillary tubes and are reported as uncorrected. TLC was carried out on precoated plates (silica gel $60 \mathrm{~F}_{254}$ ), and the spots were visualized with UV and fluorescent lights. Column chromatography was performed on silica gel (60-120 or 230-400 mesh). NMR spectra for all the compounds were recorded at 200/400/600 and 50/100/150 MHz (Bruker AVANCE 200, Bruker Ultrashield ${ }^{\mathrm{TM}}$ 400, Ascend ${ }^{\mathrm{TM}}$ 
600), respectively. IR spectra were recorded with a Perkin-Elmer FTIR instrument using a KBr pellet. HRMS spectra were obtained on XEVO-G2QTOF machine. The phrase "usual work-up" or "worked up in the usual manner" refers to washing of the organic phase with water $(2 \times 1 / 4$ of the volume of the organic phase) and brine $\left(1 \times 1 / 4\right.$ of the volume of the organic phase), drying $\left(\mathrm{Na}_{2} \mathrm{SO}_{4}\right)$, filtration, and concentration under reduced pressure.

Methyl 2-hydroxy-5-(2-methylallyloxy)benzoate (15). To a stirred solution of methyl 2,5-dihydroxybenzoate $(4 \mathrm{~g}, 23.8 \mathrm{mmol})$ in acetone $(10 \mathrm{~mL})$ potassium carbonate $(3.2 \mathrm{~g}, 23.8 \mathrm{mmol})$ was added and stirred for $15 \mathrm{~min}$. To this mixture methylallyl bromide $(3.6 \mathrm{~mL}, 35.7 \mathrm{mmol})$ was added and stirred for $2.5 \mathrm{~h}$. After the completion of the reaction, the mixture was concentrated under vacuum to remove excess acetone. The reaction mixture was then diluted with water and extracted with ethyl acetate $(2 \times 100 \mathrm{~mL})$. The combined extracts were washed successively with water $(50 \mathrm{~mL})$, saturated aqueous solution of sodium thiosulfate $(20 \mathrm{~mL})$, brine $(50$ $\mathrm{mL}$ ), dried (anhydrous $\mathrm{Na}_{2} \mathrm{SO}_{4}$ ), filtered and concentrated under reduced pressure. Column chromatography of the residue afforded ester 15 as oil (4.5 gm, 87\%). $R_{\mathrm{f}}\left(1: 5 \mathrm{EA}\right.$ (ethyl acetate)/hexane) $0.75 ;{ }^{1} \mathrm{H} \mathrm{NMR}(400 \mathrm{MHz}$, $\left.\mathrm{CDCl}_{3}\right): \delta 10.38(\mathrm{~s}, 1 \mathrm{H}), 7.31(\mathrm{~d}, J 3.2 \mathrm{~Hz}, 1 \mathrm{H}), 7.11(\mathrm{dd}, J 3.2,8.8 \mathrm{~Hz}, 1 \mathrm{H}), 6.91(\mathrm{~d}, J 8.8 \mathrm{~Hz}, 1 \mathrm{H}), 5.09(\mathrm{~s}, 1 \mathrm{H}), 4.99$ $(\mathrm{s}, 1 \mathrm{H}), 4.39(\mathrm{~s}, 2 \mathrm{H}), 3.95(\mathrm{~s}, 3 \mathrm{H}), 1.83(\mathrm{~s}, 3 \mathrm{H}) ;{ }^{13} \mathrm{C} \mathrm{NMR}\left(100 \mathrm{MHz}, \mathrm{CDCl}_{3}\right): \delta 170.3,156.1,151.1,140.8,124.7$, 118.5, 113.3, 112.9, 11.9, 72.5, 52.4, 19.5; IR (KBr): v 3220, 2954, 2362, 1683, 1488, 1218, $771 \mathrm{~cm}^{-1}$; HRMS (ESI): $m / z$ calculated for $\mathrm{C}_{11} \mathrm{H}_{11} \mathrm{O}_{3}$ : requires: 191.0708 for $[\mathrm{M}-\mathrm{OMe}]^{+}$; found: 191.0706 .

Methyl 3,6-dihydroxy-2-(2-methylprop-2-enyl)benzoate (16). Compound 15 (4 g, $18.0 \mathrm{mmol}$ ) was dissolved in DMF $(7 \mathrm{~mL})$ and heated at reflux for $10 \mathrm{~h}$. After completion of the reaction the reaction mixture was diluted with water $(10 \mathrm{~mL})$ and extracted with ethyl acetate $(2 \times 100 \mathrm{~mL})$. The combined extracts were washed successively with water $(50 \mathrm{~mL})$, brine $(50 \mathrm{~mL})$, dried (anhydrous $\mathrm{Na}_{2} \mathrm{SO}_{4}$ ), filtered and concentrated under reduced pressure. Column chromatography of the residue afforded ester $\mathbf{1 6}$ as white solid but in impure form. Since this compound was susceptible to aerial decomposition and could not be purified, it was directly subjected to methylation. $R_{\mathrm{f}}\left(1: 3 \mathrm{EA} /\right.$ hexane) $0.4 ; \mathrm{mp} 80{ }^{\circ} \mathrm{C} ;{ }^{1} \mathrm{H} \mathrm{NMR}\left(400 \mathrm{MHz}, \mathrm{CDCl}_{3}\right): \delta 10.39(\mathrm{~s}, 1 \mathrm{H}), 7.01(\mathrm{~d}$, J $8.8 \mathrm{~Hz}, 1 \mathrm{H}), 6.91(\mathrm{~d}, J 8.8 \mathrm{~Hz}, 1 \mathrm{H}), 4.83(\mathrm{~s}, 1 \mathrm{H}), 4.53(\mathrm{~s}, 1 \mathrm{H}), 3.95(\mathrm{~s}, 3 \mathrm{H}), 3.68(\mathrm{~s}, 2 \mathrm{H}), 1.82(\mathrm{~s}, 3 \mathrm{H}) ;{ }^{13} \mathrm{C} \mathrm{NMR}(100$ $\left.\mathrm{MHz}_{2} \mathrm{CDCl}_{3}\right): \delta 171.3,156.2,147.6,144.4,126.3,123.6,116.8,113.2,110.6,52.3,36.1,23.0 ; \mathrm{IR}(\mathrm{KBr}): \tilde{\mathrm{v}} 3496$, 2954, 1681, 1497, 1178, 1027, 890, $825 \mathrm{~cm}^{-1}$; HRMS (ESI): $\mathrm{m} / z$ calculated for $\mathrm{C}_{11} \mathrm{H}_{11} \mathrm{O}_{3}$ : requires: 191.0708 for $[\mathrm{M}-\mathrm{OMe}]^{+}$; found: 191.0709 .

Methyl 3,6-dimethoxy-2-(2-methylprop-2-enyl)benzoate (19). Dihydroxy compound 16 (0.67 g, $3.0 \mathrm{mmol})$ was dissolved in dry acetone $(20 \mathrm{~mL})$ under $\mathrm{N}_{2}$-atmosphere. To this solution were added dry $\mathrm{K}_{2} \mathrm{CO}_{3}(2.1 \mathrm{~g}, 15$ $\mathrm{mmol}$ ) and $\mathrm{Me}_{2} \mathrm{SO}_{4}\left(0.57 \mathrm{~mL}, 6 \mathrm{mmol}\right.$; freshly washed with cold water $(10 \mathrm{~mL})$, saturated $\mathrm{NaHCO}_{3}$ solution (15 $\mathrm{mL})$, brine $\left(15 \mathrm{~mL}\right.$ ) and dried over anhydrous $\left.\mathrm{K}_{2} \mathrm{CO}_{3}\right)$. After $2 \mathrm{~h}$ of reflux, on completion of the reaction, the inorganic salts were filtered and the filtrate was concentrated. The residue was diluted with ethyl acetate (15 $\mathrm{mL})$, treated with $\mathrm{Et}_{3} \mathrm{~N}(6 \mathrm{mmol})$ at room temperature and stirred for $30 \mathrm{~min}$. The reaction mixture was then diluted with ethyl acetate $(50 \mathrm{~mL})$, washed with water $(15 \mathrm{~mL})$ and $5 \%$ aq. $\mathrm{HCl}$ solution $(15 \mathrm{~mL})$ then subjected to usual work-up (drying over anhydrous $\mathrm{Na}_{2} \mathrm{SO}_{4}$ and concentrating under reduced pressure) to obtain a crude residue. The crude product was purified by column chromatography on silica gel to furnish pure oil 19 in $36 \%$ yield over two steps. $R_{\mathrm{f}}\left(1: 3 \mathrm{EA} /\right.$ hexane) $0.6 ;{ }^{1} \mathrm{H} \mathrm{NMR}\left(400 \mathrm{MHz}, \mathrm{CDCl}_{3}\right): \delta 6.86(\mathrm{~d}, J 8.8 \mathrm{~Hz}, 1 \mathrm{H}), 6.76(\mathrm{~d}, J 8.8$ $\mathrm{Hz}, 1 \mathrm{H}), 4.72(\mathrm{~s}, 1 \mathrm{H}), 4.48(\mathrm{~s}, 1 \mathrm{H}), 3.85(\mathrm{~s}, 3 \mathrm{H}), 3.78(\mathrm{~s}, 3 \mathrm{H}), 3.77(\mathrm{~s}, 3 \mathrm{H}), 3.34(\mathrm{~s}, 2 \mathrm{H}), 1.70(\mathrm{~s}, 3 \mathrm{H}) ;{ }^{13} \mathrm{C}$ NMR $(100$ $\left.\mathrm{MHz}, \mathrm{CDCl}_{3}\right): \delta 168.2,151.8,150.1,143.7,127.1,125.4,112.4,110.7,109.7,56.3,56.3,52.0,34.6,22.6 ; \mathrm{IR}$ $(\mathrm{KBr}): \tilde{v} 2925,1733,1560,1457,1263,1066,773 \mathrm{~cm}^{-1}$; HRMS (ESI): $\mathrm{m} / z$ calculated for $\mathrm{C}_{13} \mathrm{H}_{15} \mathrm{O}_{3}$ : requires: 219.1021 for [M-OMe] ${ }^{+}$; found: 219.1016. 
5,8-Dihydroxy-3,3-dimethylisochroman-1-one (17). Compound 17 was obtained along with 16 in $40 \%$ yield as a white solid from 15 as described above. $R_{\mathrm{f}}(1: 3 \mathrm{EA} / \mathrm{hexane})$ 0.37; IR (KBr): v 3307, 2364, 1646, 1479, 1226, 1114, 883, 819, $686 \mathrm{~cm}^{-1} ;{ }^{1} \mathrm{H}$ NMR $\left(400 \mathrm{MHz}_{\mathrm{CDCl}}\right): \delta 10.74(\mathrm{~s}, 1 \mathrm{H}), 6.99(\mathrm{~d}, J 8.8 \mathrm{~Hz}, 1 \mathrm{H}), 6.79(\mathrm{~d}, J 8.8 \mathrm{~Hz}$, $1 \mathrm{H}), 2.98(\mathrm{~s}, 2 \mathrm{H}), 1.50(\mathrm{~s}, 6 \mathrm{H}) ;{ }^{13} \mathrm{C} N M R\left(100 \mathrm{MHz} \mathrm{CDCl}_{3}\right): \delta 169.5,156.2,144.4,124.3,123.4,116.0,107.9$, 82.3, 32.9, 27.6, 27.6; HRMS (ESI): $\mathrm{m} / z$ calculated for $\mathrm{C}_{11} \mathrm{H}_{13} \mathrm{O}_{4}$ : requires: 209.0814 for $[\mathrm{M}+\mathrm{H}]^{+}$; found: 209.0812.

8-Hydroxy-3,3-dimethyl-1-oxoisochroman-5-yl acetate (18). $\mathrm{Et}_{3} \mathrm{~N}$ (0.06 mL, $0.77 \mathrm{mmol}$ ) was added to a solution of $17(80 \mathrm{mg}, 0.38 \mathrm{mmol})$ in dry $\mathrm{CH}_{2} \mathrm{Cl}_{2}(3 \mathrm{~mL})$ and stirred for $10 \mathrm{~min}$. Acetyl chloride $(0.05 \mathrm{~mL}, 0.77$ mmol) was drop-wise added to this mixture at $0{ }^{\circ} \mathrm{C}$ and allowed to stir for $2.5 \mathrm{~h}$ at room temperature. The reaction was quenched with water $(5 \mathrm{~mL})$ and extracted with $\mathrm{CH}_{2} \mathrm{Cl}_{2}(50 \mathrm{~mL})$. The combined extracts were successively washed with water $(10 \mathrm{~mL})$, brine $(10 \mathrm{~mL})$, dried (anhydrous $\left.\mathrm{Na}_{2} \mathrm{SO}_{4}\right)$, filtered and concentrated under reduced pressure. Column chromatography of the residue afforded 18 as a white solid $(80 \mathrm{mg}, 83 \%)$. $R_{\mathrm{f}}$ (1:3 EA/hexane) 0.4; mp $92{ }^{\circ} \mathrm{C} ;{ }^{1} \mathrm{H}$ NMR $\left(400 \mathrm{MHz}, \mathrm{CDCl}_{3}\right): \delta 11.11(\mathrm{~s}, 1 \mathrm{H}), 7.19(\mathrm{~d}, J 8.8 \mathrm{~Hz}, 1 \mathrm{H}), 6.91(\mathrm{~d}, J 8.8$ $\mathrm{Hz}, 1 \mathrm{H}), 2.81(\mathrm{~s}, 2 \mathrm{H}), 2.32(\mathrm{~s}, 3 \mathrm{H}), 1.48(\mathrm{~s}, 6 \mathrm{H}) ;{ }^{13} \mathrm{C} \mathrm{NMR}\left(100 \mathrm{MHz}, \mathrm{CDCl}_{3}\right): \delta 169.1,168.8,160.0,139.4,130.2$, 129.8, 116.7, 107.7, 82.0, 33.6, 27.3, 27.3, 20.6; IR (KBr): ṽ 3448, 2985, 1756, 1670, 1475, 1226, 1112, 902,804 $\mathrm{cm}^{-1}$.

[3,6-Dimethoxy-2-(2-methylprop-2-enyl)phenyl]methanol (20). ${ }^{25}$ To a stirred solution of 19 (1.0 gm, $\left.4 \mathrm{mmol}\right)$ in dry ether $(7 \mathrm{~mL})$ under nitrogen atmosphere, cooled to $0^{\circ} \mathrm{C}$, lithium aluminium hydride $(380 \mathrm{mg}, 10 \mathrm{mmol})$ was added and stirred for $3 \mathrm{~h}$ at room temperature. After completion of the reaction, it was cooled and quenched by dropwise addition of saturated sodium sulfate until a white precipitate separated. This was filtered through celite and concentrated under reduced pressure. Column chromatography of the residue afforded pure 20 (700 mg, 79\%) as colorless liquid. $R_{\mathrm{f}}(1: 1 \mathrm{EA} / \mathrm{hexane}) 0.5 ;{ }^{1} \mathrm{H} \mathrm{NMR}\left(400 \mathrm{MHz}, \mathrm{CDCl}_{3}\right): \delta 6.81$ $(\mathrm{d}, J 8.8 \mathrm{~Hz}, 1 \mathrm{H}), 6.76(\mathrm{~d}, J 8.8 \mathrm{~Hz}, 1 \mathrm{H}), 4.74(\mathrm{~s}, 1 \mathrm{H}), 4.65(\mathrm{~s}, 2 \mathrm{H}), 4.33(\mathrm{~s}, 1 \mathrm{H}), 3.84(\mathrm{~s}, 3 \mathrm{H}), 3.78(\mathrm{~s}, 3 \mathrm{H}), 3.46(\mathrm{~s}$, $2 \mathrm{H}), 1.81(\mathrm{~s}, 3 \mathrm{H})$.

4-Methoxy-3-(2-methallyloxy)benzaldehyde (21). ${ }^{26}$ To a stirred solution of isovanillin ( $\left.8.8 \mathrm{~g}, 57.9 \mathrm{mmol}\right)$ in dry acetone $(300 \mathrm{~mL})$ was added solid $\mathrm{K}_{2} \mathrm{CO}_{3}(8.0 \mathrm{~g}, 57.9 \mathrm{mmol})$ at $0{ }^{\circ} \mathrm{C}$ followed by addition of methallyl bromide $(8.8 \mathrm{~mL}, 86.9 \mathrm{mmol})$. The reaction mixture was stirred for $24 \mathrm{~h}$ and filtered. The filtrate was concentrated and extracted with ethyl acetate $(250 \mathrm{~mL})$. Organic layer was washed with $\mathrm{H}_{2} \mathrm{O}(3 \times 100 \mathrm{~mL})$ and brine $(100 \mathrm{~mL})$. Combined organic layer was dried over $\mathrm{Na}_{2} \mathrm{SO}_{4}$ and evaporated under vacuum to afford the crude material. It was purified by performing flash column chromatography on silica gel with 1:5 ethyl acetate/hexane solvent to afford 21 (10.9 g, $52.7 \mathrm{mmol}$ ) in 91\% yield. Yellow oil; ${ }^{1} \mathrm{H} \mathrm{NMR}\left(200 \mathrm{MHz}, \mathrm{CDCl}_{3}\right) \delta 9.75(\mathrm{~s}, 1 \mathrm{H}), 7.41-7.32(\mathrm{~m}$, $2 \mathrm{H}), 6.91(\mathrm{~d}, J 8.2 \mathrm{~Hz}, 1 \mathrm{H}), 5.06(\mathrm{~s}, 1 \mathrm{H}), 4.94(\mathrm{~s}, 1 \mathrm{H}), 4.49(\mathrm{~s}, 2 \mathrm{H}), 3.88(\mathrm{~s}, 3 \mathrm{H}), 1.77(\mathrm{~s}, 3 \mathrm{H}) ;{ }^{13} \mathrm{C} \mathrm{NMR}(50 \mathrm{MHz}$, $\left.\mathrm{CDCl}_{3}\right) \delta 190.8(\mathrm{CH}), 154.9,148.7,140.1,130.0,126.7(\mathrm{CH}), 113.2\left(\mathrm{CH}_{2}\right), 111.2,110.8(\mathrm{CH}), 72.5\left(\mathrm{CH}_{2}\right), 56.1$ $\left(\mathrm{CH}_{3}\right), 19.3\left(\mathrm{CH}_{3}\right) ; \mathrm{IR}(\mathrm{KBr}): \tilde{v}=3422,2936,1686,1611,1582,1508,1438,1270,1220,1162,1134,1017,905$, $642 \mathrm{~cm}^{-1}$.

Compound 21 (1.1 g, $5.33 \mathrm{mmol})$ was dissolved in DMF $(5 \mathrm{~mL})$ or 1, 2-dichlorobenzene and heated to reflux at $250{ }^{\circ} \mathrm{C}$. Expected product was not obtained. From 1, 2-dichlorobenzene, products were $22(0.245 \mathrm{~g}, 1.3 \mathrm{mmol}$, 25\%), 23 (0.200 g, $1.00 \mathrm{mmol}, 19 \%)$. From DMF, yields were 22 (0.035 g, $0.19 \mathrm{mmol}, 4 \%), 23$ (0.070 g, 0.37 $\mathrm{mmol}, 7 \%), 24$ (0.255 g, $1.24 \mathrm{mmol}, 23 \%)$.

2-Methoxy-7-methylnaphthalen-1-ol (22). Yellow liquid; ${ }^{1} \mathrm{H}$ NMR (200 MHz, CDCl$) \delta 8.06(d, J 0.6,1 \mathrm{H}), 7.73$ (d, J $8.4 \mathrm{~Hz}, 1 \mathrm{H}), 7.42(\mathrm{~d}, J$ 9.0, 1H), $7.28(\mathrm{~d}, \mathrm{~d}, J 8.4,1.6 \mathrm{~Hz}, 1 \mathrm{H}), 7.21(\mathrm{~d}, J 9.0 \mathrm{~Hz}, 1 \mathrm{H}), 6.24(\mathrm{bs}, 1 \mathrm{H}), 3.97(\mathrm{~s}, 3 \mathrm{H})$, $2.60(\mathrm{~s}, 3 \mathrm{H}) ;{ }^{13} \mathrm{C} \mathrm{NMR}\left(100 \mathrm{MHz}, \mathrm{CDCl}_{3}\right): \delta 141.5,139.3,135.2,128.1,127.5(\mathrm{CH}), 126.8(\mathrm{CH}), 124.3,120.1(\mathrm{CH})$, $119.5(\mathrm{CH}), 112.4(\mathrm{CH}), 57.1\left(\mathrm{CH}_{3}\right), 22.0\left(\mathrm{CH}_{3}\right) ; \mathrm{IR}(\mathrm{KBr}): \tilde{v} 3422,2927,1741,1608,1459,1275,1220,1051,678$ 
$\mathrm{cm}^{-1}$. HRMS (ESI): $\mathrm{m} / \mathrm{z}$ calculated for $\mathrm{C}_{12} \mathrm{H}_{12} \mathrm{O}_{2}$ : requires: 187.0759 for $[\mathrm{M}-\mathrm{H}]+, 188.0837$ for [M], 189.0916 $[\mathrm{M}+\mathrm{H}], 191.1072$ [M+3H]; found: 187.0749, 188.0837, 189.0893, 191.1068.

2-Methoxy-7-methyl-7,8-dihydronaphthalen-1-ol (23). Yellow liquid; ${ }^{1} \mathrm{H} \mathrm{NMR}\left(200 \mathrm{MHz}, \mathrm{CDCl}_{3}\right) \delta 6.66$ (d, J 8.0 $\mathrm{Hz}, 1 \mathrm{H}), 6.58(\mathrm{~d}, J 8.0 \mathrm{~Hz}, 1 \mathrm{H}), 6.35(\mathrm{dd}, J 2.8,9.6 \mathrm{~Hz}, 1 \mathrm{H}), 5.70(\mathrm{~s}, 1 \mathrm{H}), 3.88(\mathrm{~s}, 3 \mathrm{H}), 3.06-3.01(\mathrm{~m}, 1 \mathrm{H}), 2.56-2.51$ $(\mathrm{m}, 1 \mathrm{H}), 2.47-2.41(\mathrm{~m}, 1 \mathrm{H}), 1.13(\mathrm{~d}, J 6.8 \mathrm{~Hz}, 3 \mathrm{H}) ;{ }^{13} \mathrm{C} \mathrm{NMR}\left(50 \mathrm{MHz}, \mathrm{CDCl}_{3}\right): \delta 146.1,142.6,133.2(\mathrm{CH}), 128.2$, $126.5(\mathrm{CH}), 120.7,117.5(\mathrm{CH}), 107.9(\mathrm{CH}), 56.2\left(\mathrm{CH}_{3}\right), 28.6\left(\mathrm{CH}_{2}\right), 28.4(\mathrm{CH}), 20.7\left(\mathrm{CH}_{3}\right)$; IR (KBr): $\tilde{\mathrm{v}}$ 3454, 2956, 1636, 1491, 1275, 1079, 806, $772 \mathrm{~cm}^{-1}$; HRMS (ESI): $\mathrm{m} / z$ calculated for $\mathrm{C}_{12} \mathrm{H}_{14} \mathrm{O}_{2}$ : requires: 191.1072 for $[\mathrm{M}+\mathrm{H}]+$; found: 191.1085 .

2-Methoxy-7-methyl-7,8-dihydro-naphthalen-1-yl acetate. ${ }^{1} \mathrm{H} \mathrm{NMR}\left(600 \mathrm{MHz}, \mathrm{CDCl}_{3}\right) \delta 6.88(\mathrm{~d}, J 8.3 \mathrm{~Hz}, 1 \mathrm{H})$, $6.73(\mathrm{~d}, J 8.2 \mathrm{~Hz}, 1 \mathrm{H}), 6.35$ (dd, J 2.2, $9.6 \mathrm{~Hz}, 1 \mathrm{H}), 5.75(\mathrm{dd}, J 3.3,9.8 \mathrm{~Hz}, 1 \mathrm{H}), 3.81(\mathrm{~s}, 3 \mathrm{H}), 2.77(\mathrm{dd}, J 6.6,15.7$ $\mathrm{Hz}, 1 \mathrm{H}), 2.51$ (qd, J 3.4, $6.8 \mathrm{~Hz}, 1 \mathrm{H}), 2.35(\mathrm{~s}, 4 \mathrm{H}), 1.09(\mathrm{~d}, J 7.1 \mathrm{~Hz}, 3 \mathrm{H}) .{ }^{13} \mathrm{C} \mathrm{NMR}\left(150 \mathrm{MHz}, \mathrm{CDCl}_{3}\right) \delta 169.1$, $150.6,137.5,133.1,128.7,127.8,126.1,123.9,109.4,56.1,29.6,28.3,20.7,20.5$. IR (KBr): ṽ 2921, 2851, 1764, $1463,1275,1219,1191,1077 \mathrm{~cm}^{-1}$. HRMS (ESI): $\mathrm{m} / \mathrm{z}$ calculated for $\mathrm{C}_{14} \mathrm{H}_{16} \mathrm{O}_{3}$ : requires: $233.1178 \mathrm{for}[\mathrm{M}+\mathrm{H}]+$; found: 233.1193 .

7-Methoxy-2,2-dimethyl-2,3-dihydrobenzofuran-4-carbaldehyde (24). ${ }^{27}{ }^{1} \mathrm{H} \mathrm{NMR}\left(200 \mathrm{MHz}, \mathrm{CDCl}_{3}\right) \delta 9.85(\mathrm{~s}$, $1 \mathrm{H}), 7.27(\mathrm{~d}, J 8.4 \mathrm{~Hz}, 1 \mathrm{H}), 6.83(\mathrm{~d}, J 8.4 \mathrm{~Hz}, 1 \mathrm{H}), 3.91(\mathrm{~s}, 3 \mathrm{H}), 3.35(\mathrm{~s}, 2 \mathrm{H}), 1.49(\mathrm{~s}, 6 \mathrm{H}) ;{ }^{13} \mathrm{C} \mathrm{NMR}(50 \mathrm{MHz}$, $\left.\mathrm{CDCl}_{3}\right): \delta 191.6(\mathrm{CH}), 149.5,148.2,128.6,127.3(\mathrm{CH}), 126.9,110.4(\mathrm{CH}), 89.3,56.2\left(\mathrm{CH}_{3}\right), 43.4\left(\mathrm{CH}_{2}\right), 28.5(2 \mathrm{x}$ $\mathrm{CH}_{3}$ ); IR (KBr): v 2972, 1686, 1611, 1582, 1508, 1438, 1270, 1220, 1162, 1134, 1017, 905, $642 \mathrm{~cm}^{-1}$; HRMS (ESI): $\mathrm{m} / \mathrm{z}$ calculated for $\mathrm{C}_{12} \mathrm{H}_{14} \mathrm{O}_{3}$ : requires: 207.1021 for $[\mathrm{M}+\mathrm{H}]+$; found: 207.1038 .

[3,6-Dimethoxy-2-(2-methallyl)-phenyl]methanol (20). ${ }^{25}$ To a stirred solution of compound 28 (0.44 g, 2 $\mathrm{mmol})$ in THF $(10 \mathrm{~mL})$ and $\mathrm{MeOH}(3 \mathrm{~mL})$ at $0{ }^{\circ} \mathrm{C}$ was added $\mathrm{NaBH}_{4}(84 \mathrm{mg}, 2.2 \mathrm{mmol})$ in portions. The resulting mixture was stirred at $r$ for overnight. Then it was quenched with saturated $\mathrm{NH}_{4} \mathrm{Cl}(3 \mathrm{~mL})$. Extraction of the reaction mixture with $\mathrm{EA}(3 \times 10 \mathrm{~mL})$ followed by drying over $\mathrm{Na}_{2} \mathrm{SO}_{4}$ and removal of solvent gave a residue. Flash column chromatography of the residue on silica gel with 1:2 EA/hexane solvent afforded product 20 $(0.38 \mathrm{~g}, 1.72 \mathrm{mmol})$ as colorless liquid in $86 \%$ yield.

2-Hydroxymethyl-3-(2-methylprop-2-enyl)-1,4-benzoquinone (14). To a stirred solution of 20 (280 mg, 1.26 $\mathrm{mmol}$ ) in acetonitrile $(7 \mathrm{~mL})$, solution of cerric ammonium nitrate $(2.07 \mathrm{gm}, 3.78 \mathrm{mmol})$ in water $(7 \mathrm{~mL}) \mathrm{was}$ added drop-wise and stirred for $1 \mathrm{~h}$. Then the reaction mixture was extracted with ethyl acetate $(2 \times 50 \mathrm{~mL})$. The combined extracts were washed successively with water $(25 \mathrm{~mL})$, brine $(25 \mathrm{~mL})$, dried (anhydrous $\mathrm{Na}_{2} \mathrm{SO}_{4}$ ), filtered and concentrated under reduced pressure. Column chromatography of the residue afforded 14 (160 $\mathrm{mg}, 66 \%)$. Yellow solid; $R_{\mathrm{f}}\left(1: 1 \mathrm{EA} /\right.$ hexane) $0.4 ;{ }^{1} \mathrm{H} \mathrm{NMR}\left(400 \mathrm{MHz}, \mathrm{CDCl}_{3}\right): \delta 6.74(\mathrm{~d}, J 10 \mathrm{~Hz}, 1 \mathrm{H}), 6.70(\mathrm{~d}, J 10$ $\mathrm{Hz}, 1 \mathrm{H}), 4.73(\mathrm{~s}, 1 \mathrm{H}), 4.47(\mathrm{~s}, 2 \mathrm{H}), 4.45(\mathrm{~s}, 1 \mathrm{H}), 3.21(\mathrm{~s}, 2 \mathrm{H}), 2.46$ (brs, $1 \mathrm{H}), 1.71(\mathrm{~s}, 3 \mathrm{H}) ;{ }^{13} \mathrm{C} \mathrm{NMR}(100 \mathrm{MHz}$, $\left.\mathrm{CDCl}_{3}\right): \delta 188.7,187.0,142.8,142.2,141.9,136.8(\mathrm{CH}), 136.3(\mathrm{CH}), 111.9\left(\mathrm{CH}_{2}\right), 57.4\left(\mathrm{CH}_{2}\right), 32.7\left(\mathrm{CH}_{2}\right), 23.2$ $\left(\mathrm{CH}_{3}\right)$; IR (KBr): $\tilde{v} 3448,2933,1654,1297,1018,850 \mathrm{~cm}^{-1}$.

2-(2-Methylprop-2-enyl)-3-(tetrahydropyran-2-yloxymethyl)-1,4-benzoquinone (29). To a stirred solution of $14(40 \mathrm{mg}, 0.21 \mathrm{mmol})$ in dry dichloromethane $(5 \mathrm{~mL})$ were added dihydropyran $(0.04 \mathrm{~mL}, 0.42 \mathrm{mmol})$ and a catalytic amount of PPTS and stirred for $3 \mathrm{~h}$. Then the reaction mixture was extracted with ethyl acetate (50 $\mathrm{mL})$. The combined extracts were washed successively with water $(10 \mathrm{~mL})$, brine $(10 \mathrm{~mL})$, dried (anhydrous $\left.\mathrm{Na}_{2} \mathrm{SO}_{4}\right)$, filtered and concentrated under reduced pressure. Column chromatography of the residue afforded 29 (55 mg, 96\%). Yellow semisolid; $R_{\mathrm{f}}\left(1: 3 \mathrm{EA} /\right.$ hexane) 0.65; ${ }^{1} \mathrm{H} \mathrm{NMR}\left(400 \mathrm{MHz}, \mathrm{CDCl}_{3}\right): \delta 6.78(\mathrm{~s}, 2 \mathrm{H}), 4.79(\mathrm{~s}$, $1 \mathrm{H}), 4.60(\mathrm{~d}, J 10.8 \mathrm{~Hz}, 1 \mathrm{H}), 4.52(\mathrm{~s}, 1 \mathrm{H}), 4.33(\mathrm{~d}, J 10.8 \mathrm{~Hz}, 1 \mathrm{H}), 3.87(\mathrm{t}, J 9.6 \mathrm{~Hz}, 1 \mathrm{H}), 3.40-3.60(\mathrm{~m}, 2 \mathrm{H}), 3.34(\mathrm{~s}$, $2 \mathrm{H}), 1.80$ (s, 3H), 1.40-1.79 (m, 6H); IR (KBr): ṽ 2942, 1656, 1440, 1297, 1120, 1025, $771 \mathrm{~cm}^{-1} ; \mathrm{HRMS}$ (ESI): $m / z$ calculated for $\mathrm{C}_{16} \mathrm{H}_{20} \mathrm{O}_{4}$ : requires: 175.0759 for [M-OTHP]+; found: 175.0761 . 
1,4-Dihydroxy-2-(2-methylprop-2-enyl)-3-(tetrahydropyran-2-yloxymethyl) anthraquinone (32). A solution of 30 (242 mg, $1 \mathrm{mmol})$ in dry THF ( $5 \mathrm{~mL}$ ) was added to suspension of LiOBu-t $(240 \mathrm{mg}, 3 \mathrm{mmol})$ in dry THF at -60 ${ }^{\circ} \mathrm{C}$ under an inert atmosphere. The resulting solution was stirred at $-60{ }^{\circ} \mathrm{C}$ for $30 \mathrm{~min}$ after which a solution of 29 (331 mg, $1.2 \mathrm{mmol}$ ) in dry THF $(5 \mathrm{~mL})$ was added to it. The reaction was stirred for another $30 \mathrm{~min}$ at $-60{ }^{\circ} \mathrm{C}$ followed by $6-8 \mathrm{~h}$ at room temperature. The reaction was then quenched with saturated ammonium chloride solution and THF was removed under reduced pressure. The residue was then extracted with ethyl acetate ( 3 $x 20 \mathrm{~mL})$. The combined extracts were washed with brine $\left(3 \times 1 / 3\right.$ vol), dried $\left(\mathrm{Na}_{2} \mathrm{SO}_{4}\right)$ and concentrated to provide crude product. The crude product was purified by column chromatography to furnish quinone 32 . Red solid; $R_{\mathrm{f}}\left(1: 3 \mathrm{EA} /\right.$ hexane) $0.7 ; \mathrm{mp} 125-127{ }^{\circ} \mathrm{C} ;{ }^{1} \mathrm{H}$ NMR $\left(400 \mathrm{MHz}, \mathrm{CDCl}_{3}\right): \delta 13.62(\mathrm{~s}, 1 \mathrm{H}), 13.50(\mathrm{~s}, 1 \mathrm{H}), 8.34-$ $8.40(\mathrm{~m}, 2 \mathrm{H}), 7.70-7.90(\mathrm{~m}, 2 \mathrm{H}), 4.88(\mathrm{~d}, J 10.8 \mathrm{~Hz}, 1 \mathrm{H}), 4.80(\mathrm{~s}, 1 \mathrm{H}), 4.65(\mathrm{~d}, J 10.8 \mathrm{~Hz}, 1 \mathrm{H}), 4.39(\mathrm{~s}, 1 \mathrm{H}), 3.98(\mathrm{t}$, J $8.4 \mathrm{~Hz}, 1 \mathrm{H}), 3.67(\mathrm{~s}, 2 \mathrm{H}), 3.50-3.65(\mathrm{~m}, 2 \mathrm{H}), 1.89(\mathrm{~s}, 3 \mathrm{H}), 1.50-1.83(\mathrm{~m}, 6 \mathrm{H}) ;{ }^{13} \mathrm{C} \mathrm{NMR}\left(100 \mathrm{MHz}^{\mathrm{C}} \mathrm{CDCl}\right): \delta$ 186.9, 186.5, 157.5, 156.8, 143.1, 142.2, 137.1, $134.3(\mathrm{CH}), 134.2(\mathrm{CH}), 133.6,133.5,126.9(\mathrm{CH}), 126.9(\mathrm{CH})$, 111.8, 111.1, $110.9\left(\mathrm{CH}_{2}\right), 98.9(\mathrm{CH}), 62.2\left(\mathrm{CH}_{2}\right), 59.4\left(\mathrm{CH}_{2}\right), 33.5\left(\mathrm{CH}_{2}\right), 30.5\left(\mathrm{CH}_{2}\right), 25.4\left(\mathrm{CH}_{2}\right), 23.4\left(\mathrm{CH}_{3}\right), 19.3$ $\left(\mathrm{CH}_{2}\right)$; IR (KBr): $\tilde{\mathrm{v}} 2927,2372,1623,1401,1265,1024,773 \mathrm{~cm}^{-1}$. HRMS (ESI): $\mathrm{m} / z$ calculated for $\mathrm{C}_{24} \mathrm{H}_{24} \mathrm{O}_{6}$ : requires: 431.1471 for $[\mathrm{M}+\mathrm{Na}]+, 409.1651$ for $[\mathrm{M}+\mathrm{H}], 391.1546$ for $\left[\mathrm{M}-\mathrm{H}_{2} \mathrm{O}+\mathrm{H}\right]+, 325.1076$ for $[\mathrm{M}-\mathrm{DHP}+\mathrm{H}]+$, 307.0970 for [M-DHP- $\left.\mathrm{H}_{2} \mathrm{O}+\mathrm{H}\right]+$; found: 431.1483, 409.1667, 391.1545, 325.1082, 307.0992.

1,4-Dihydroxy-2-hydroxymethyl-3-(2-methylprop-2-enyl)anthraquinone (31). To a stirred solution of 32 (100 $\mathrm{mg}, 0.25 \mathrm{mmol})$ in methanol $(5 \mathrm{~mL})$, a catalytic amount of PPTS was added and stirred for $1 \mathrm{~h}$. Then the reaction mixture was extracted with ethyl acetate $(50 \mathrm{~mL})$. The combined extracts were washed successively with water $(10 \mathrm{~mL})$, brine $(10 \mathrm{~mL})$, dried (anhydrous $\left.\mathrm{Na}_{2} \mathrm{SO}_{4}\right)$, filtered and concentrated under reduced pressure. Column chromatography of the residue afforded quinone 31 (60 mg, 76\%). Red solid; mp 117-118 ${ }^{\circ} \mathrm{C} ; R_{\mathrm{f}}(1: 3 \mathrm{EA} / \mathrm{hexane}) 0.6 ;{ }^{1} \mathrm{H} \mathrm{NMR}\left(400 \mathrm{MHz}, \mathrm{CDCl}_{3}\right): \delta 13.68(\mathrm{~s}, 1 \mathrm{H}), 13.51(\mathrm{~s}, 1 \mathrm{H}), 8.35-8.40(\mathrm{~m}, 2 \mathrm{H}), 7.90-$ $7.70(\mathrm{~m}, 2 \mathrm{H}), 4.83(\mathrm{~s}, 1 \mathrm{H}), 4.79(\mathrm{~s}, 2 \mathrm{H}), 4.45(\mathrm{~s}, 1 \mathrm{H}), 3.63(\mathrm{~s}, 2 \mathrm{H}), 2.70$ (brs, $1 \mathrm{H}), 1.88(\mathrm{~s}, 3 \mathrm{H}) ;{ }^{13} \mathrm{C} \mathrm{NMR}(100 \mathrm{MHz}$, $\left.\mathrm{CDCl}_{3}\right): \delta 186.9,186.9,157.0,156.9,143.7,139.9,139.6,139.6,134.6(\mathrm{CH}), 134.5(\mathrm{CH}), 133.6,133.5,127.2$ $(\mathrm{CH}), 127.0(\mathrm{CH}), 111.5,111.4\left(\mathrm{CH}_{2}\right), 57.3\left(\mathrm{CH}_{2}\right), 33.4\left(\mathrm{CH}_{2}\right), 23.3\left(\mathrm{CH}_{3}\right) ; \mathrm{IR}(\mathrm{KBr}): \tilde{v} 3426,2923,2370,1587$, 1401, 1263, 1020, $798 \mathrm{~cm}^{-1}$; HRMS (ESI): $\mathrm{m} / z$ calculated for $\mathrm{C}_{19} \mathrm{H}_{16} \mathrm{O}_{5}$ : requires: 325.1076 for $[\mathrm{M}+\mathrm{H}]+, 307.0970$ for $\left[\mathrm{M}-\mathrm{H}_{2} \mathrm{O}+\mathrm{H}\right]+$; found: 325.1082, 307.0956.

1,4-Dihydroxy-3-(2-methyl-allyl)-9,10-dioxo-9,10-dihydroanthracene-2-carboxaldehyde (33). PCC (213 mg, $0.99 \mathrm{mmol}$ ) was added to a stirred solution of $\mathbf{3 1}(108 \mathrm{mg}, 0.33 \mathrm{mmol})$ in DCM (5 mL) and stirring was continued for $3 \mathrm{~h}$ at rt. Solvent was evaporated and slurried with basic alumina. Column chromatography was done with basic alumina and 1:20 ethyl acetate/haxane solvent. Compound 33 (73 mg, 0.23 mmol) was obtained in $69 \%$ yield. Yellow solid; $\mathrm{mp} 100{ }^{\circ} \mathrm{C} ; R_{\mathrm{f}}\left(1: 1 \mathrm{EA} /\right.$ hexane) $0.7 ;{ }^{1} \mathrm{H} \mathrm{NMR}\left(400 \mathrm{MHz}, \mathrm{CDCl}_{3}\right): \delta 13.69(\mathrm{~s}$, $1 \mathrm{H}), 13.27(\mathrm{~s}, 1 \mathrm{H}), 10.68(\mathrm{~s}, 1 \mathrm{H}), 8.41-8.30(\mathrm{~m}, 2 \mathrm{H}), 7.90-7.86(\mathrm{~m}, 2 \mathrm{H}), 4.75(\mathrm{~s}, 1 \mathrm{H}), 4.33(\mathrm{~s}, 1 \mathrm{H}), 3.88(\mathrm{~s}, 2 \mathrm{H})$, 1.90 (s, 3H). ); ${ }^{13} \mathrm{C}$ NMR $\left(100 \mathrm{MHz}, \mathrm{CDCl}_{3}\right): \delta 190.6(\mathrm{CH}), 186.7,186.5,159.3,155.8,143.7,143.3,134.9(\mathrm{CH})$, $134.8(\mathrm{CH}), 133.0,133.0,131.0,131.0,127.3(\mathrm{CH}), 127.0(\mathrm{CH}), 112.0,110.4\left(\mathrm{CH}_{2}\right), 32.6\left(\mathrm{CH}_{2}\right), 23.6\left(\mathrm{CH}_{3}\right) ; \mathrm{IR}$ (KBr): $\tilde{v} 3375,2372,1585,1218,1052,771 \mathrm{~cm}^{-1}$. HRMS (ESI): $\mathrm{m} / z$ calculated for $\mathrm{C}_{19} \mathrm{H}_{14} \mathrm{O}_{5}$ : requires: 322.0841 for $[\mathrm{M}]+, 321.0763$ for $[\mathrm{M}-\mathrm{H}]+, 293.0814$ for $[\mathrm{M}-\mathrm{HCHO}+\mathrm{H}]+$; found: 322.0809, 321.0775, 293.0820.

6,7,11-Trihydroxy-9-methylene-7,8,9,10-tetrahydronaphthalene-5,12-dione (11). A stirred solution of 33 $(0.145 \mathrm{~g}, 0.45 \mathrm{mmol})$ in dry DCM $(4.5 \mathrm{~mL})$ was treated with $\mathrm{SnCl}_{4} \cdot 5 \mathrm{H}_{2} \mathrm{O}(0.079 \mathrm{~g}, 0.23 \mathrm{mmol})$ in stoppered reaction flask. After completion of the reaction, as monitored by TLC, it was quenched with saturated solution of sodium bicarbonate $(2.5 \mathrm{~mL} / \mathrm{mmol})$. The reaction mixture was then extracted with DCM $(3 \times 5 \mathrm{~mL})$. The combined extracts were washed with brine $\left(3 \times 1 / 3\right.$ vol), dried $\left(\mathrm{Na}_{2} \mathrm{SO}_{4}\right)$ and concentrated to provide crude product. After flash column chromatography by silica gel with 1:2 ethyl acetate/hexane solvent compound $\mathbf{1 1}$ ( $0.129 \mathrm{~g}, 0.40 \mathrm{mmol})$ was obtained in $89 \%$ yield. $R_{\mathrm{f}}\left(1: 1 \mathrm{EA} /\right.$ hexane) $0.65 ;{ }^{1} \mathrm{H} \mathrm{NMR}\left(400 \mathrm{MHz}, \mathrm{CDCl}_{3}\right): \delta 13.72(\mathrm{~s}$, 
1H), 13.37 (s, 1H), 8.40-8.32 (m, 2H), 7.80-7.88 (m, 2H), 5.30 (brs, 1H), $5.16(\mathrm{~s}, 1 \mathrm{H}), 5.12(\mathrm{~s}, 1 \mathrm{H}), 3.63(\mathrm{ABd}, J$ 20.4, 2H), 3.52 (ABd, J $20.4 \mathrm{~Hz}, 2 \mathrm{H}), 2.91$ (brs, $1 \mathrm{H}), 2.71$ (d, J $4.4 \mathrm{~Hz}, 2 \mathrm{H}) ;{ }^{13} \mathrm{C} \mathrm{NMR}\left(100 \mathrm{MHz}, \mathrm{CDCl}_{3}\right): \delta 186.8$, 186.7, 156.6, 156.0, 138.8, 138.3, 137.7, $134.4(\mathrm{CH}), 134.4(\mathrm{CH}), 133.5,133.4,127.0(\mathrm{CH}), 127.0(\mathrm{CH}), 113.4$ $\left(\mathrm{CH}_{2}\right), 110.5,63.8,38.7,32.1$ (one quaternary carbon is missing); IR (KBr): v 3450, 2370, 1583, 1403, 1222, $1024 \mathrm{~cm}^{-1}$; HRMS (ESI): $\mathrm{m} / z$ calculated for $\mathrm{C}_{19} \mathrm{H}_{14} \mathrm{O}_{5}$ : requires: 323.0920 for $[\mathrm{M}+\mathrm{H}]+$; found: 323.0925.

Dihydroxylation of 34. To a stirred solution of compound $34(192 \mathrm{mg}, 1.2 \mathrm{mmol})$ in THF (36 mL) and $\mathrm{H}_{2} \mathrm{O}(18$ $\mathrm{mL}$ ) were added $\mathrm{OsO}_{4}(2.4 \mathrm{~mL} 0.05 \mathrm{M}$ solution in toluene, $0.12 \mathrm{mmol}$ ) and $\mathrm{NMO}(422 \mathrm{mg}, 3.6 \mathrm{mmol})$. The reaction vessel was wrapped by a black paper and stoppered well. Reaction mixture was stirred for $15 \mathrm{~h}$ at $\mathrm{rt}$. After the starting material disappeared, the reaction was quenched by adding sodium bisulfite. Then usual work-up was done with ethyl acetate. Crude material was subjected to column chromatography packed with basic alumina to give product 35 (149 mg, $0.77 \mathrm{mmol}, 64 \%$ ), 36 (35 mg, $0.18 \mathrm{mmol}, 15 \%$ ).

2,4-Dihydroxy-1,2,3,4-tetrahydronaphthalen-2-ylmethyl-oxonium (35). White solid; mp $98-100{ }^{\circ} \mathrm{C} ; R_{\mathrm{f}}(2: 1$ EA/hexane) 0.3; ${ }^{1} \mathrm{H}$ NMR (400 MHz, $\left.\mathrm{CDCl}_{3}\right): \delta 7.47(\mathrm{~d}, J 7.2 \mathrm{~Hz}, 1 \mathrm{H}), 7.22-7.28(\mathrm{~m}, 2 \mathrm{H}), 5.04(\mathrm{t}, J 5.4 \mathrm{~Hz}, 1 \mathrm{H})$, $3.68(\mathrm{~d}, J 11.2 \mathrm{~Hz}, 1 \mathrm{H}), 3.55$ (d, J $11.2 \mathrm{~Hz}, 1 \mathrm{H}), 2.92$ (d, J $16.8 \mathrm{~Hz}, 2 \mathrm{H}), 2.85$ (d, J $16.8 \mathrm{~Hz}, 2 \mathrm{H}), 2.35$ (bs, $2 \mathrm{H}), 2.21$ $(\mathrm{dd}, J 5.2,13.6 \mathrm{~Hz}, 1 \mathrm{H}), 2.05$ (bs, $1 \mathrm{H}), 1.97(\mathrm{dd}, J 6.8,13.6 \mathrm{~Hz}, 1 \mathrm{H}) ;{ }^{1} \mathrm{H}$ NMR $\left(400 \mathrm{MHz}\right.$, acetone- $\left.d_{6}\right): \delta 7.55(\mathrm{~d}, J$ $7.2 \mathrm{~Hz}, 1 \mathrm{H}), 7.10-7.17(\mathrm{~m}, 2 \mathrm{H}), 7.04(\mathrm{~d}, J 7.6 \mathrm{~Hz}, 1 \mathrm{H}), 4.96(\mathrm{~d}, J 3.6,1 \mathrm{H}), 4.28(\mathrm{~s}, 1 \mathrm{H}), 3.98(\mathrm{bs}, 1 \mathrm{H}), 3.59(\mathrm{bs}, 1 \mathrm{H})$, $3.59(\mathrm{~s}, 1 \mathrm{H}), 3.46(\mathrm{~s}, 2 \mathrm{H}), 2.94(\mathrm{~s}, 2 \mathrm{H}), 2.68(\mathrm{~d}, J 16.8,1 \mathrm{H}), 2.15-2.20(\mathrm{~m}, 1 \mathrm{H}), 1.69-1.75(\mathrm{~m}, 1 \mathrm{H}) ;{ }^{13} \mathrm{C} \mathrm{NMR}(100$ $\mathrm{MHz}$, acetone- $\left.d_{6}\right): \delta 141.5,135.6,129.6(\mathrm{CH}), 127.5(\mathrm{CH}), 127.5(\mathrm{CH}), 126.5(\mathrm{CH}), 72.7,71.2\left(\mathrm{CH}_{2}\right), 67.2(\mathrm{CH})$, $41.8\left(\mathrm{CH}_{2}\right), 39.3\left(\mathrm{CH}_{2}\right)$; IR (KBr): $\tilde{\mathrm{v}} 3398,2920,1637,1220,1066 \mathrm{~cm}^{-1}$; HRMS (ESI): $\mathrm{m} / z$ calculated for $\mathrm{C}_{11} \mathrm{H}_{14} \mathrm{O}_{3}$ : requires: 129.0704 for $\left[\mathrm{M}-\mathrm{CH}_{3} \mathrm{OH}-\mathrm{H}_{2} \mathrm{O}_{2}+\mathrm{H}\right]+, 141.0704$ for $\left[\mathrm{M}-3 \mathrm{H}_{2} \mathrm{O}+\mathrm{H}\right]+, 159.0810$ for $\left[\mathrm{M}-2 \mathrm{H}_{2} \mathrm{O}+\mathrm{H}\right]+, 217.0841$ for [M+Na]+; found: 129.733, 141.0746, 159.0839, 217.0913.

3-Hydroxy-3-hydroxymethyl-3,4-dihydro-2H-naphthalen-1-one (36). White solid; $\mathrm{mp} 43-45{ }^{\circ} \mathrm{C} ; R_{\mathrm{f}}(1: 2$ EA/hexane) 0.4; ${ }^{1} \mathrm{H}$ NMR (400 MHz, $\mathrm{CDCl}_{3}$ ): $\delta 8.03(\mathrm{~d}, J 7.6 \mathrm{~Hz}, 1 \mathrm{H}), 7.52(\mathrm{t}, J 7.6 \mathrm{~Hz}, 1 \mathrm{H}), 7.34(\mathrm{t}, J 7.6 \mathrm{~Hz}), 7.28$ (d, J $7.6 \mathrm{~Hz}, 1 \mathrm{H}), 3.61(\mathrm{~s}, 2 \mathrm{H}), 3.18(\mathrm{~d}, J 16.8 \mathrm{~Hz}, 1 \mathrm{H}), 3.12(\mathrm{~d}, J 16.8 \mathrm{~Hz}, 1 \mathrm{H}), 2.86(\mathrm{~d}, J 16.8 \mathrm{~Hz}, 1 \mathrm{H}), 2.75(\mathrm{~d}, J$ $16.8 \mathrm{~Hz}, 1 \mathrm{H}$ ), 2.54 (bs, $1 \mathrm{H}), 2.23$ (bs, $1 \mathrm{H}) ;{ }^{1} \mathrm{H}$ NMR (400 MHz, acetone- $\left.d_{6}\right): \delta 7.90-7.92(\mathrm{~m}, 1 \mathrm{H}), 7.50-7.54(\mathrm{~m}$, $1 \mathrm{H}), 7.29-7.32(\mathrm{~m}, 2 \mathrm{H}), 4.18(\mathrm{t}, J 5.6 \mathrm{~Hz}), 3.87(\mathrm{~s}, 1 \mathrm{H}), 3.54(\mathrm{~d}, J 5.6 \mathrm{~Hz}, 2 \mathrm{H}), 3.32(\mathrm{~d}, J 16.6 \mathrm{~Hz}, 1 \mathrm{H}), 2.98(\mathrm{~d}, J$ 16.6 Hz, 1H), $2.84(\mathrm{dd}, J 2.0,16.6 \mathrm{~Hz}, 1 \mathrm{H}), 2.64(\mathrm{dd}, J 2.0,16.6 \mathrm{~Hz}, 1 \mathrm{H}) ;{ }^{13} \mathrm{C} \mathrm{NMR}\left(100 \mathrm{MHz}\right.$, acetone- $\left.d_{6}\right): \delta 197.4$, 142.6, $134.3(\mathrm{CH}), 133.4,130.8(\mathrm{CH}), 127.1(\mathrm{CH}), 126.8(\mathrm{CH}), 74.8,70.2\left(\mathrm{CH}_{2}\right), 48.1\left(\mathrm{CH}_{2}\right), 39.0\left(\mathrm{CH}_{2}\right) ; \mathrm{IR}(\mathrm{KBr}): \tilde{\mathrm{v}}$ 3422, 2921, 1636, $1220 \mathrm{~cm}^{-1}$; HRMS (ESI): $\mathrm{m} / z$ calculated for $\mathrm{C}_{11} \mathrm{H}_{12} \mathrm{O}_{3}$ : requires: 175.0759 for $\left[\mathrm{M}-\mathrm{H}_{2} \mathrm{O}+\mathrm{H}\right]+$, 193.0865 for $[\mathrm{M}+\mathrm{H}]+$; found: $175.0786,193.0891$.

4-(tert-Butyl-dimethylsilanyloxy)-3,4-dihydro-1H-naphthalen-2-one (40). ${ }^{30} \mathrm{~A}$ stream of ozone was passed through a stirred solution of $39(550 \mathrm{mg}, 2.0 \mathrm{mmol})$ in dry DCM $(10 \mathrm{~mL})$ at $-78{ }^{\circ} \mathrm{C}$ until resulting solution became blue-violet. The excess ozone was removed by passing a stream of oxygen followed by argon through the solution. The resulting mixture was allowed to reach $-50{ }^{\circ} \mathrm{C}$ and carefully quenched by addition of $\mathrm{Ph}_{3} \mathrm{P}$ $(630 \mathrm{mg}, 2.4 \mathrm{mmol})$ in dry DCM $(2.5 \mathrm{~mL})$. After $24 \mathrm{~h}$ at $\mathrm{rt}$ the reaction mixture was concentrated and the residue was subjected to chromatography on basic alumina and eluted with 1:30 ethyl acetate/hexane solvent. Product 40 (359 mg, $1.3 \mathrm{mmol}$ ) was obtained in $65 \%$ yield. Yellow liquid; $R_{\mathrm{f}}(1: 20 \mathrm{EA} / \mathrm{hexane}) 0.5 ;{ }^{1} \mathrm{H}$

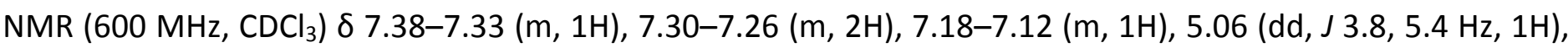
3.77 (dd, 1H, J 1.0, 19.7 Hz), $3.51(\mathrm{~d}, J 19.8 \mathrm{~Hz}, 1 \mathrm{H}), 2.75-2.63(\mathrm{~m}, 2 \mathrm{H}), 0.86(\mathrm{~s}, 9 \mathrm{H}), 0.13(\mathrm{~s}, 3 \mathrm{H}),-0.05(\mathrm{~s}, 3 \mathrm{H})$; ${ }^{13} \mathrm{C} \mathrm{NMR}\left(150 \mathrm{MHz}, \mathrm{CDCl}_{3}\right) \delta 208.6,139.2,133.1,128.8(\mathrm{CH}), 128.4(\mathrm{CH}), 127.1(\mathrm{CH}), 126.5(\mathrm{CH}), 70.0(\mathrm{CH})$, $48.0\left(\mathrm{CH}_{2}\right), 44.6\left(\mathrm{CH}_{2}\right), 25.9\left(3 \times \mathrm{CH}_{3}\right), 18.3,-4.5\left(\mathrm{CH}_{3}\right),-4.6\left(\mathrm{CH}_{3}\right) ; \mathrm{IR}(\mathrm{KBr}): \tilde{\mathrm{v}} 2930,2857,1702,1256,1219$, 1081, 952, 836, $670 \mathrm{~cm}^{-1}$. HRMS (ESI): $\mathrm{m} / z$ calculated for $\mathrm{C}_{16} \mathrm{H}_{24} \mathrm{O}_{2} \mathrm{Si}$ : requires: 277.1624 for [M+H]+ ; found: 277.1636. 


\section{Supplementary Materials}

Analytical data $\left({ }^{1} \mathrm{H} N M R,{ }^{13} \mathrm{C}\right.$ NMR, single crystal X-ray diffraction) for all new compounds.

\section{Acknowledgements}

We gratefully acknowledge the Council of Scientific and Industrial Research (CSIR), New Delhi for financial support, and the Department of Science and Technology, New Delhi for NMR instrumental facilities. S. B. and S. R. thank CSIR for their research fellowships.

\section{References}

1. Snider, B. B. Acc. Chem. Res. 1980, 13, 426-432.

https://doi.org/10.1021/ar50155a007

2. Clarke, M. L.; France, M. B. Tetrahedron 2008, 64, 9003-9031 and references therein. https://doi.org/10.1016/j.tet.2008.06.075

3. Mikami, K.; Shimizu, M. Chem. Rev. 1992, 92, 1021-1050.

https://doi.org/10.1021/cr00013a014

4. Oppolzer, W.; Snieckus, V. Angew. Chem. Int. Ed. Engl. 1978, 17, 476-486.

https://doi.org/10.1002/anie.197804761

5. Johnson, J. S.; Evans, D. A. Acc. Chem. Res. 2000, 33, 325-335.

https://doi.org/10.1021/ar960062n

6. Trost, B. M.; Li, Y. J. Am. Chem. Soc. 1996, 118, 6625-6633.

https://doi.org/10.1021/ja960642f

7. Pitts, M. R.; Mulzer, J. Tetrahedron Lett. 2002, 43, 8471-8473.

https://doi.org/10.1016/S0040-4039(02)02086-5

8. Evans, D. A.; Kværnø, L.; Dunn, T. B.; Beauchemin, A.; Raymer, B.; Mulder, J. A.; Olhava, E. J.; Juhl, M.; Kagechika, K.; Favor, D. A. J. Am. Chem. Soc. 2008, 130, 16295-16309.

https://doi.org/10.1021/ja804659n

9. Hauser, F. M.; Mal, D. J. Am. Chem. Soc. 1984, 106, 1862-1863.

https://doi.org/10.1021/ja00318a065

10. Hauser, F. M.; Mal, D. J. Am. Chem. Soc. 1984, 106, 1098-1104.

https://doi.org/10.1021/ja00316a048

11. Hauser, F. M.; Mal, D. J. Am. Chem. Soc. 1983, 105, 5688-5690.

https://doi.org/10.1021/ja00355a028

12. Hauser, F. M.; Hewawasam, P.; Mal, D. J. Am. Chem. Soc. 1988, 110, 2919-2924.

https://doi.org/10.1021/ja00217a038

13. Hauser, F. M.; Hewawasam, P. J. Org. Chem. 1988, 53, 4515-4519. Brown, E. G.; Cambie, R. C.; Holroyd, S. E.; Johnson, M.; Rutledge, P. S.; Woodgate, P. D. Tetrahedron. Lett. 1989, 30, 4735-4736.

14. Combie, R. C.; Higgs, K. C.; Rustenhoven, J. J.; Rutledge, P. S. Aust. J. Chem. 1996, 49, 677-688.

15. Combie, R. C.; Higgs, K. C.; Rustenhoven, J. J.; Rutledge, P. S. Aust. J. Chem. 1996, 49, 751-759.

16. Wong, C. M.; Popien, D.; Schenk, Raa, J. T. Can. J. Chem. 1971, 49, 2712-2718. 
17. Sallam, M. A. E.; Whistler, R. L.; Cassady, J. M. Can. J. Chem. 1985, 63, 2697-2702.

\section{https://doi.org/10.1139/v85-448}

18. Basak, S.; Mal, D. Tetrahedron 2016, 72, 1758-1772.

https://doi.org/10.1016/j.tet.2016.02.033

19. Binaschi, M.; Bigioni, M.; Cipollone, A.; Rossi, C.; Goso, C.; Maggi, C. A.; Capranico, G.; Animati, F. Curr. Med. Chem.-Anti-Cancer Agents 2001, 1, 113-130.

https://doi.org/10.2174/1568011013354723

20. Arcamone, F. M. Biochimie 1998, 80, 201-206.

https://doi.org/10.1016/S0300-9084(98)80003-5

21. Krohn, K. Anthracycline Chemistry and Biology I: Biological Occurrence and Biosynthesis, Synthesis and Chemistry; Topics in Current Chemistry; Springer 1 edition: Berlin/Heidelberg, 2008.

22. Fang, L.; Zhang, G.; Li, C.; Zheng, X.; Zhu, L.; Xiao, J. J.; Szakacs, G.; Nadas, J.; Chan, K. K.; Wang, P. G.; Sun, D. J. Med. Chem. 2006, 49, 932-941.

https://doi.org/10.1021/jm050800q

23. Combie, R. C.; Clark, R. B.; Rustenhoven, J. J.; Rutledge, P. S. Aust. J. Chem. 1999, 52, 781-800. https://doi.org/10.1071/CH99014

24. Mal, D.; Ray, S.; Sharma, I. J. Org. Chem. 2007, 72, 4981-4984. https://doi.org/10.1021/jo062271j

25. Koning, C. B. D.; Green, I. R.; Michael, J. P.; Oliveira, J. R. Tetrahedron 2001, 57, 9623-9634. https://doi.org/10.1016/S0040-4020(01)00963-2

26. Jagdale, A. R.; Park, J. H.; Youn, S. W. J. Org. Chem., 2011, 76, 7204-7215. https://doi.org/10.1021/jo201339z

27. Ohshima, E.; Kawakita, T.; Yanagawa, K.; lida, K.; Koike, R.; Nakasato, Y.; Matsuzaki, T.; Ohmori, K.; Sato, K. S.; Ishii, H. U.S. Pat. Appl. Publ., US 20020128290 A1 Sep 12, 2002.

28. Mal, D.; J. Teach. Res. Chem. 1997, 4, 16-19.

29. Mal, D.; Ray, S. Eur. J. Org. Chem. 2008, 17, 3014-3020. https://doi.org/10.1002/ejoc.200800218

30. Boltukhina, E. V.; Sheshenev, A. E.; Lyapkalo, I. M. Tetrahedron 2011, 67, 5382-5388. https://doi.org/10.1016/i.tet.2011.05.095 